\title{
A low-cost instrumentation approach for seismic hazard assessment in urban areas
}

\author{
C. Z. Karakostas ${ }^{1}$ \& V. K. Papanikolaou ${ }^{2}$ \\ ${ }^{I}$ Earthquake Planning and Protection Organization \\ (EPPO-ITSAK), Greece \\ ${ }^{2}$ Department of Civil Engineering, \\ Aristotle University of Thessaloniki, Greece
}

\begin{abstract}
The objective of the present study is to propose a low-cost instrumentation methodology for urban areas, in order to effectively record actual strong motion variations on an adequately dense grid during at least light intensity seismic events. The proposed methodology is an efficient alternative to usual microzonation studies, which, due to economic reasons, are typically based on relatively few actual recordings and then use theoretical assumptions to assess the strong motion distribution throughout the area under study. The availability of actual recordings on a dense grid, which is feasible to implement through this new approach, minimizes many theoretical uncertainties inherent in the usual studies. For this purpose, an autonomous triaxial accelerograph based on MEMS sensor technology has been designed and manufactured, together with custom developed software for device configuration and data retrieval. The production cost of the developed devices is an order of magnitude lower than the presently available commercial products, thus making economically feasible their deployment on a dense grid all over the urban area under study. As a pilot case, over 20 prototype accelerographs have been already installed on a high-seismicity urban area in Greece, aiming to record the local variability of strong motion within a relatively small area. The expected strong motion records are georeferenced and postprocessed with custom designed GIS-type software, in order to produce explicit seismic hazard maps of the considered urban area. The application of the above procedure requires minimum maintenance and human intervention at a fraction of the cost compared to available commercial solutions. Keywords: MEMS, accelerograph, instrumentation, hazard maps.
\end{abstract}




\section{Introduction}

The development of microzonation studies for seismic hazard assessment stemmed from the early recognition by the scientific community (e.g. Seed and Schnabel [1]) of the fact that in case of an earthquake, significant variations in the ground motion can be observed within small distances in an urban area, depending on local geological and geophysical conditions. Hence, for the design of important structures, or for urban development planning, the adoption of the single seismic hazard zone typically proposed for the whole region by the national Seismic Codes is inadequate. Typically, due to high equipment cost, only a limited number - if any - of seismic recording instruments are installed throughout an urban area. The estimation of the site-specific amplification of the excitation from the sparsely available actual recordings requires the application of analytical methods that depend on the availability of expensive-to-procure data on the underlying soil and primary bedrock profile (e.g. through borehole data, measurements of shear wave velocities, standard penetration test values, micro tremor measurements etc.). However, it should be noted that the accuracy and the reliability of the above implicit procedures for estimating strong motion characteristics on the ground level are not ensured, since they highly depend on the debatable accuracy of the knowledge of the soil layer profiles throughout the studied region and the inherent uncertainties of the respective attenuation relationships and amplification assumptions that will be used. It is hence obvious that the density and quality of the actual field measurements of the seismic excitations play a predominant role in reducing uncertainties in microzonation studies and generally in seismic hazard assessment procedures. The main objective of the present study is to develop an economically feasible methodology for the deployment of low-cost seismic sensors in urban areas on an adequately dense grid, in order to effectively capture actual strong motion data during at least light intensity seismic events. This was realized by designing and producing autonomous accelerographs based on the micro-electromechanical sensor (MEMS) technology. Although use of MEMS in devices for engineering seismology and structural monitoring activities has rapidly grown in the last decade (e.g. Avery et al. [2], Kim et al. [3], Picozzi et al. [4]), the manufacturing costs vary significantly, depending on the number of embedded features (e.g. sensor accuracy, processing power, connectivity etc.).

The concept behind the present research effort was to develop low-cost accelerographs, that can be used for the dense instrumentation of urban areas, thus providing an 'explicit' solution to the various aforementioned issues which rise the cost and uncertainties of a typical microzonation study. Hence, it is inevitable that there will be an acceptable reduction in measurement accuracy compared to expensive commercial devices, but the effort was made to optimize cost-effectiveness, while not compromising the basic requirement of the present application, i.e. the accurate capturing of near field at least light intensity earthquake events (PGA larger than approx. $0.014 \mathrm{~g} \mathrm{[5]).} \mathrm{So,} \mathrm{in} \mathrm{the} \mathrm{present}$ development stage at least, and in order to keep a low commercial cost (i.e. less than $500 €$ ), no GSM/WiFi capabilities were incorporated in the devices. On the 
other hand, particular attention was given to user-friendliness and simplicity both in setting up and operating the device, as well as in data retrieval and processing. A description of the developed devices and the related software can be found in the following chapters. It should additionally be noted that the availability of a vast number of actual data from a dense instrumentation can be used, apart for the already described 'explicit' construction of seismic hazard maps, also for the proper validation and calibration of the theoretical procedures and assumptions used in regions where typical microzonation studies have already been performed. Finally it must be mentioned, that the low cost of the devices practically eliminates the need for specialised repair facilities and personnel, since it is economically feasible to just replace a defective unit with a new one.

\section{The low-cost accelerograph}

The low cost-accelerograph SeismoBug ${ }^{\odot}$ was designed and manufactured at the research unit of the Earthquake Planning and Protection Organization (EPPO-ITSAK), within a two-year post-doctorate research grant by the Greek Secretariat of Research and Technology (GSRT) (fig. 1). It is an autonomous triaxial accelerograph, based on the latest MEMS sensor technology. The device can operate totally unattended on external power supply with battery backup (load sharing), featuring automatic event triggering and non-volatile local storage (up to $2 \mathrm{~GB}$ ). It also incorporates a pre-trigger buffer memory (1 Mbit), self-calibration, accurate $(2 \mathrm{ppm})$ event timestamping, relatively low-noise output $(<2 \mathrm{mg}$ RMS at 150 SPS $)$ for its cost, and compact size $(120 \times 65 \times 40 \mathrm{~mm})$. Device configuration and data retrieval are performed via cable or Bluetooth connection to a host computer (230.4 Kbps), using custom communication software.
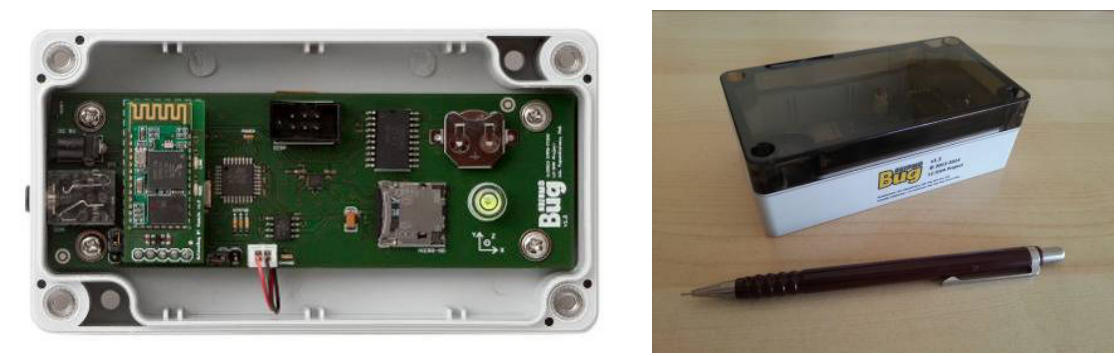

Figure 1: The low-cost accelerograph SeismoBug ${ }^{\odot}$.

The in-house developed communication software SeismoBug ${ }^{\mathcal{O}}$ Monitor (fig. 2) supports device configuration with geo-location referencing, real-time recording and advanced record post-processing capabilities. Configurable parameters include (a) device ID and map location, (b) sensor settings including sampling rate and range, (c) real-time clock settings (d) triggering settings including two different algorithms (absolute acceleration threshold and STA/LTA) and (e) pre- and post-event recording periods. Using the 
communication software, the recorded data can be quickly transferred from local storage (microSD card) to the host computer and further exported in spreadsheet format. Moreover, the device can be operated in real-time (keyboard-triggering) mode, for manual continuous recording. Finally, signal postprocessing features have been developed for easy multi-record viewing/comparison in both time and frequency domains.

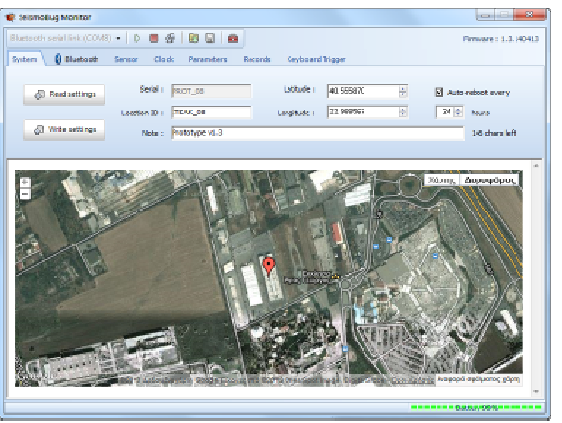

(a)

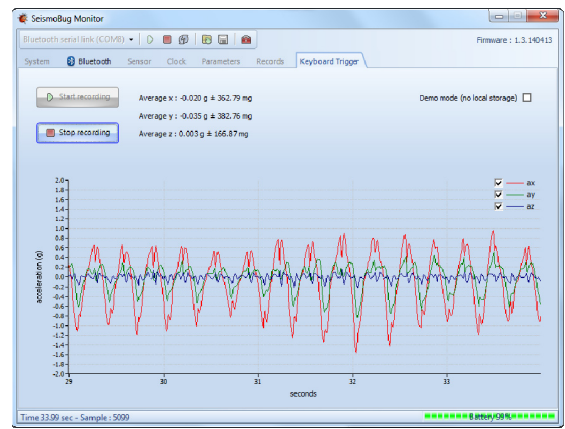

(c)

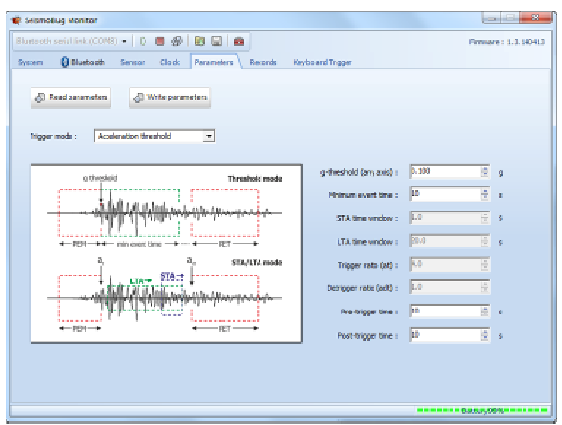

(b)

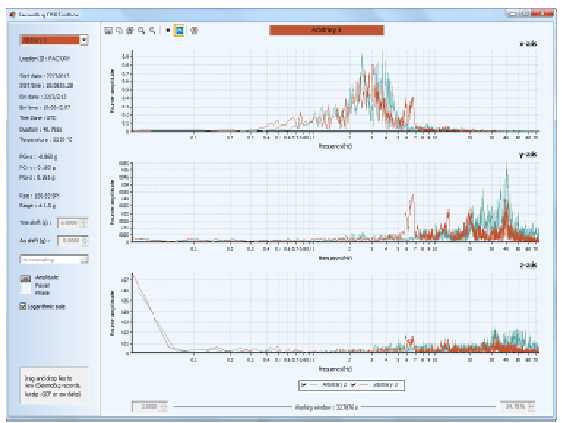

(d)

Figure 2: $\quad$ The SeismoBug ${ }^{\circ}$ Monitor software: (a) Device and location settings (b) triggering modes (c) real-time (streaming mode) view of recorded excitation (d) FFTs of recorded events.

Device validation was performed using a uniaxial seismic table at the EPPOITSAK laboratory, where the present device was mounted collinearly with a high accuracy/cost commercial accelerometer (Güralp Systems CMG-5TDE), in order to test the output accuracy both in terms of time and acceleration output. Various sinusoidal and arbitrary excitations were applied and the recorded data from both devices were compared both in time and frequency domains. The observed correlation was excellent (fig. 3) with negligible differences between the two instruments. A more detailed presentation of the device and its validation can be found in [6]. 

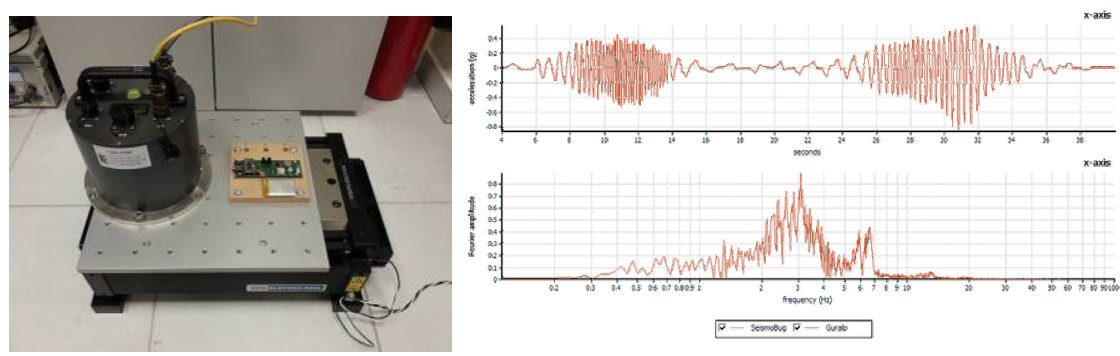

Figure 3: Seismic table setup and comparisons in time and frequency domain.

\section{Free-field application}

After the successful laboratory validation, the next step of the present research program was the testing of an adequate number of manufactured devices in real-field conditions, through a pilot instrumentation of an earthquake-prone urban area in Greece. The area selected was the city of Lefkas, capital of the Lefkas (Lefkada) island, located in the Ionian Sea. This choice was made due to the historical high seismicity of the broader Ionian region, which is classified as the highest earthquake hazard zone (design PGA $=0.36 \mathrm{~g}$ ) in the Greek Seismic Code (Eurocode 8) (fig. 4). A secondary reason was that island of Lefkas is connected by a road bridge to the continental country, which makes it easily accessible for the necessary maintenance trips for the pilot network.

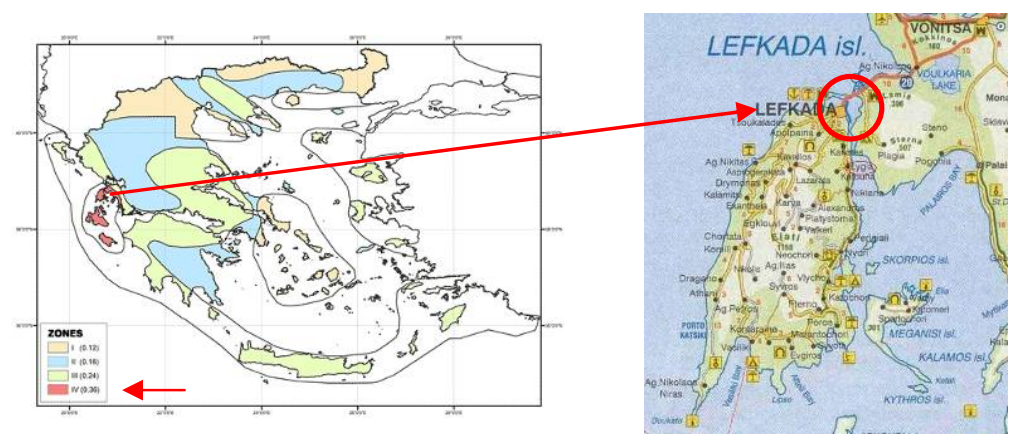

Figure 4: The selected high seismicity urban area of Lefkas city.

In order to select the installation locations, the following criteria were considered: (a) the regularity of the network grid on the broader city area, which should become more dense towards the more highly populated city centre, (b) the free-field conditions at the selected locations, which should preferably be minimally affected by nearby structures and (c) the availability of uninterrupted power supply. Under these conditions, it was decided that the most convenient solution was to install the logging units inside feeder pillars (steel enclosures) that provide electric power to street lights. The main advantage of selecting 
feeder pillars, apart from the obvious uninterruptable power supply and protection from environmental conditions, is that they are mounted on large solid concrete blocks cast directly on the ground (fig. 5). As a result, they are expected to reliably represent free-field conditions during a strong motion event. It is noted though, that in case of a power outage during such a strong event, the device automatically switches to battery power operation that can last over 100 hours.

If pillars were not available at locations of interest (in order to construct a regular network grid), or in cases where the pillar was not mounted on a concrete block, the alternative respective options were to mount the devices either on the ground slab or in the basement (if existed) of public buildings (near the centre of rotation of the load-bearing system of the structure), or to use external waterproof electrical junction boxes (fig. 6). The above procedure resulted in a total of 21 acceleration logging stations of which 10 were mounted inside feeder pillars, 4 on junction boxes, 5 on building ground slabs and 2 in basements. The primary (x) axis of the units was aligned to the North and care was taken for a firm mount and horizontal levelling of the devices.

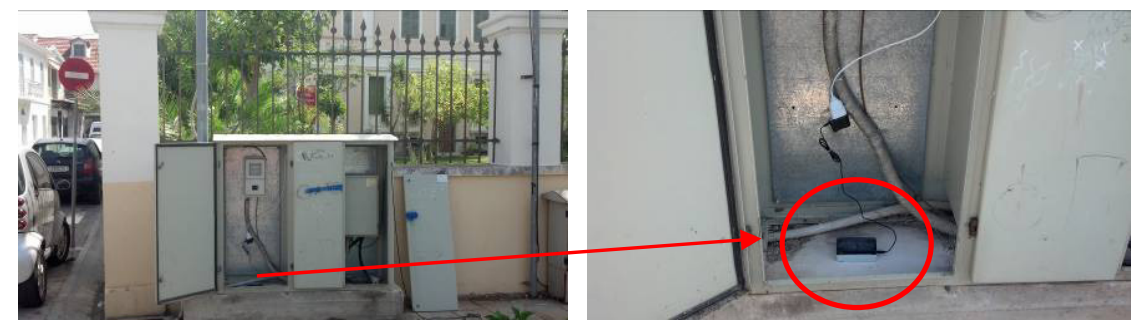

Figure 5: Device installation inside a feeder pillar.
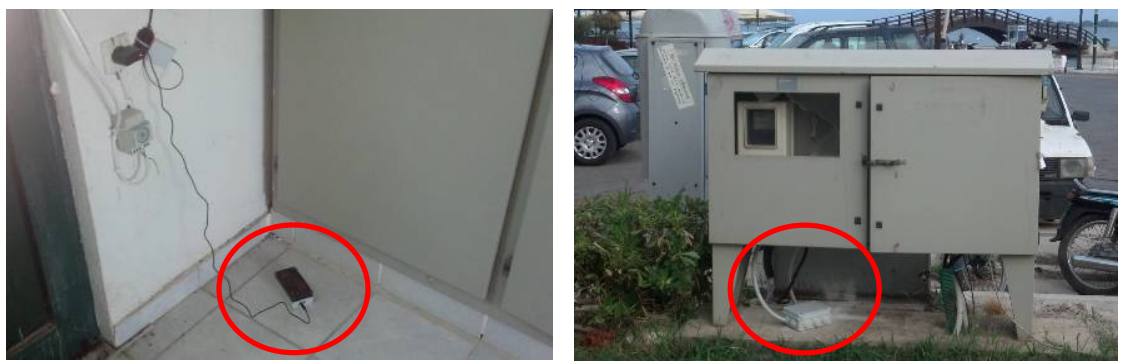

Figure 6: Device installation on a ground slab (left) and in junction box (right).

It is noted that an exception to the grid regularity rule was made for two of the above units, for a special reason. Specifically the two units were installed adjacent to two high accuracy instruments (Güralp) that belong to the National Accelerometer Network operated by EPPO-ITSAK: the first in the basement of 
the Lefkas Municipality building and the second, in free-field conditions inside a feeder pillar near the building (at a distance of approximately $70 \mathrm{~m}$ ). This setup for the Güralp accelerographs was chosen during a parallel research effort by EPPO-ITSAK in order to study the effect of the seismic response of a building housing an accelerograph on the response recorded by the instrument. The placement of the newly developed low-cost devices next to the high-accuracy ones will provide the ability to compare recorded data between the two kinds instruments in the case of a seismic event (similarly to the lab validation procedure described earlier) and to validate their applicability for similar theoretical investigations on the effect of the housing structure on the sensor recordings.

A regional map of the broader urban area of Lefkas including the installation points of all 21 logging devices is depicted in figure 8 . It is observed that the perseverance of grid normality was met, with a growing density towards the city centre. Moreover, it is noted that a single unit was deliberately installed on the opposite side of the sea canal that separates the island from the continental country, in order to provide a proper grid triangulation, essential to produce smooth seismic hazard contour maps.

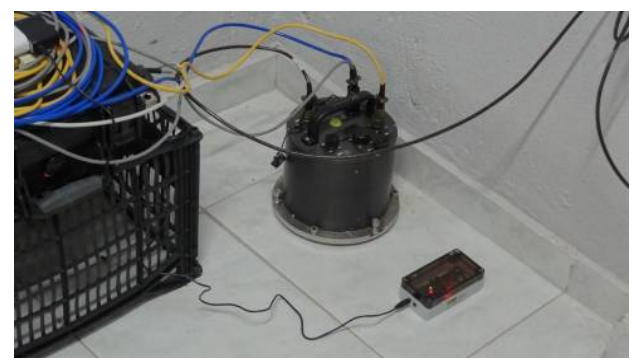

Figure 7: Device installation next to a high accuracy instrument.
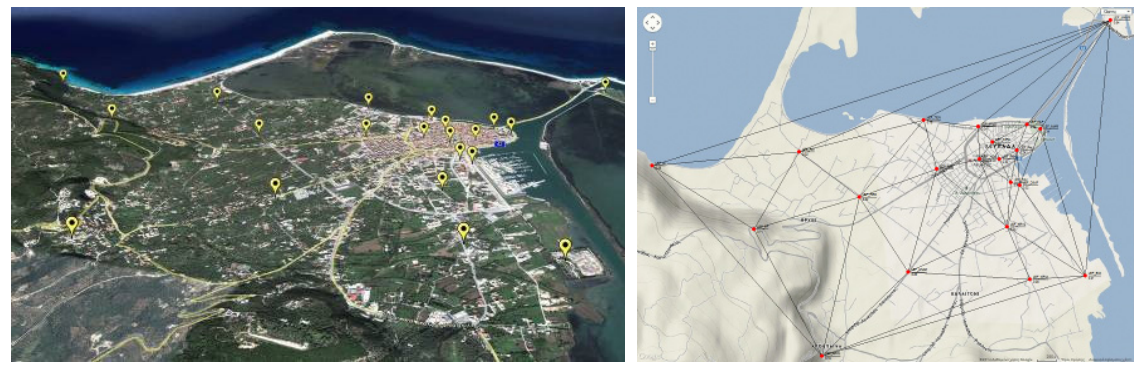

Figure 8: Accelerograph network installed on the broader urban area of the city of Lefkas. 
The installed devices were synchronised to a common time reference, within the limitations set by the inherent accuracy of their internal real time clock ( $2 \mathrm{ppm}$ - about 5 seconds per month). This issue was confirmed during regular maintenance visits; however such a small time difference between units meets the requirements of the present research effort, which is focused on the ability to unambiguously identify a 'common' seismic event. Moreover, all units were configured to use the absolute acceleration threshold triggering scheme (set for each unit from $0.02 \mathrm{~g}$ to $0.03 \mathrm{~g}$, depending on the surrounding background noise, mainly due to car traffic). This scheme is sufficient to capture at least light intensity seismic events, however it is prone to false triggering due to the inherent baseline drift ('zero-g' level) of the MEMS sensor, due to intraday temperature variations. As a result, and despite the fact that the units were programmed to automatically recalibrate every 24 hours, there were a few cases where a large number of noise signals were undesirably recorded. A possible remedy to this practical (yet not critical, due to ample available storage) problem is to apply a different triggering scheme such as the STA/LTA algorithm (already implemented in the software but requiring very elaborate, trial-and-error calibration) or RMS triggering, which has a clearer physical meaning and is still not affected by baseline drifting (soon to be incorporated in the software).

\section{Data processing and hazard maps}

After a successful recording of a strong motion event, data retrieval from the logging stations is an easy task using the wireless data transfer feature. The recorded data are stored in a text-style spreadsheet format which includes 3 timeacceleration data series for the three orthogonal axes, together with various properties such as event timestamping, duration, temperature, station name/location, sampling rate etc. In order to facilitate easy and fast browsing through the usually large amount of retrieved bulk data, the custom software SeismoBug ${ }^{\mathscr{O}}$ Browser was also developed. In figure 9, a screenshot of this program depicts the captured ground motion of an installed device due to nearby road construction works. It is noted that basic metadata are also featured (FFT, STA, LTA, RMS), for a quick visual assessment of the nature of the recorded excitation. For instance, from the figure it is clear that the recorded motion is non-seismic, since the excitation frequencies are well above the range of approximately $20 \mathrm{~Hz}$ that typically corresponds to an upper limit for earthquake excitations.

Following the selection of records of interest, these are forwarded to the dedicated GIS-type software SeismoBug ${ }^{\mathcal{O}}$ Manager for creating the seismic hazard maps. The user only has to select two folders containing records and station configuration files, respectively. The station locations are then plotted on an online map (powered by the Google $\mathrm{Maps}^{\mathcal{O}}$ service) and the various concurrent records (within a user-set time tolerance that takes into account the non-perfect synchronization between devices described in the previous section) are automatically grouped as 'common events'. Figure 10 shows a screenshot of the program which depicts the installed stations in the city of Lefkas. Since 
actual earthquake data are not yet available, a set of simulated records were used, and which were recognized by the software as pertaining to three different events, shown on the left window pane. Active stations (i.e. which participate in the selected by the user, yellow-highlighted event) are marked as red pins on the right pane.

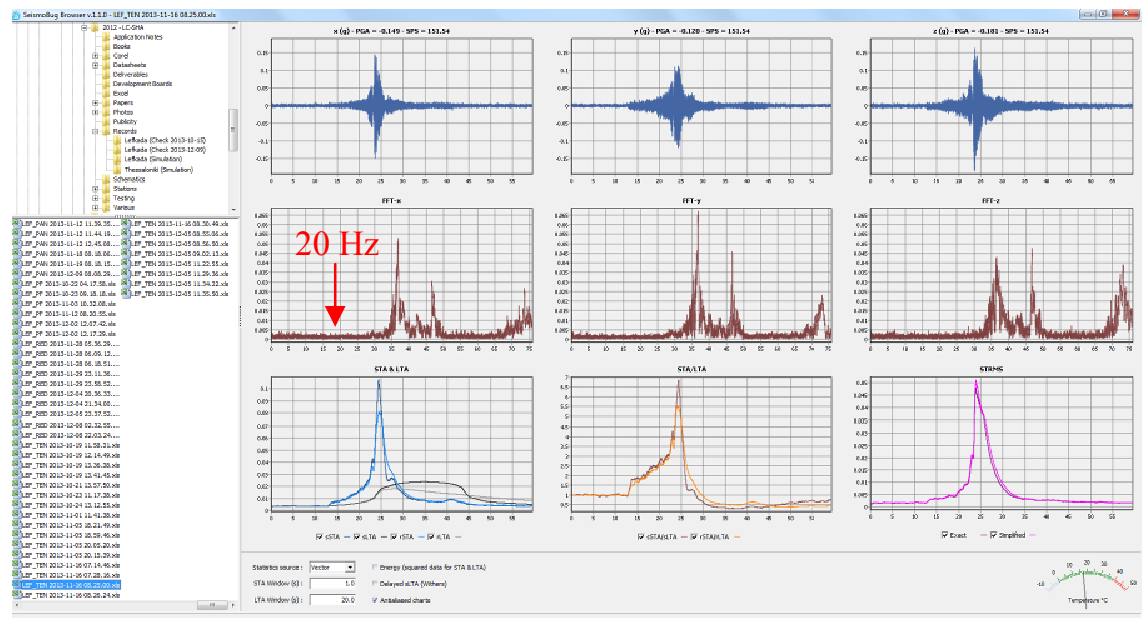

Figure 9: $\quad$ The SeismoBug ${ }^{\complement}$ Browser software.

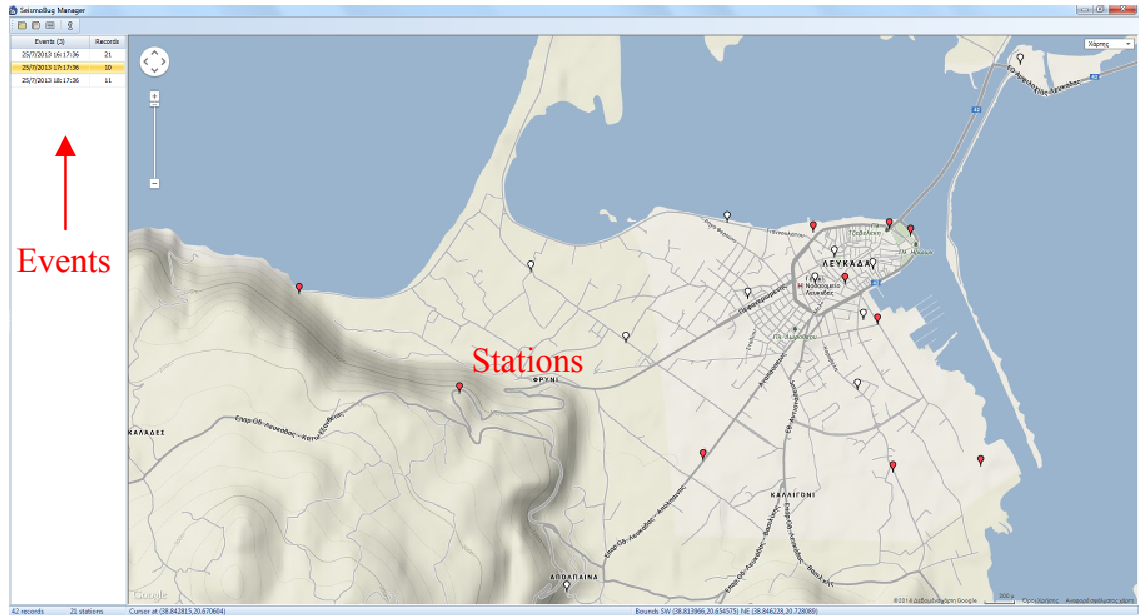

Figure 10: $\quad$ The SeismoBug ${ }^{\odot}$ Manager software: station map and event list.

With a single button click, a seamless integration with the third-party contour mapping software 3DField ${ }^{\odot}$ (http://3dfmaps.com, free for less than 50 triangulation points) is performed. A map snapshot is captured and automatically opened in 3 DField $^{\odot}$, with three PGA values $(x, y, z)$ associated to 
each station, as derived from the source strong motion record data. Using the 3DField software, the user can easily produce seismic hazard contour maps (PGA spatial distribution), as depicted in figure 11. It has to be pointed out, that due to the proximity of the recording stations which is economically feasible through the use of the developed low-cost accelerographs, the produced hazard maps are based directly on the actual recordings, without need to recourse to additional data or assumptions on underlying soil profiles, attenuation and amplification laws of propagating seismic waves etc. which are needed in typical microzonation studies. It is also noted that baseline correction and bandpass filtering of the raw acceleration data, which should be pre-applied before map creation, are soon to be integrated into the SeismoBug ${ }^{\mathcal{O}}$ Manager and Browser programs.

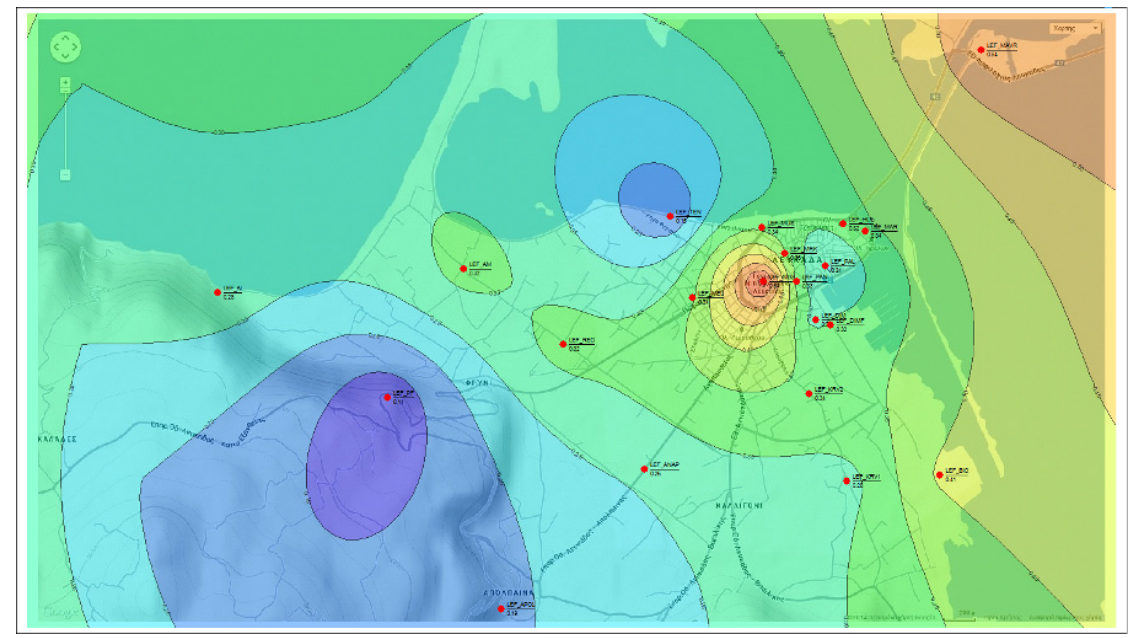

Figure 11: The SeismoBug ${ }^{\mathcal{O}}$ Manager software: seamless integration with 3DField for producing seismic hazard maps.

\section{Conclusions and further work}

In this study, an instrumentation methodology for urban areas, aiming to capture a detailed picture of the strong motion variation during at least light intensity seismic events was presented. The methodology is based on the design and installation of low-cost accelerographs, installed on an adequately dense grid in the area under consideration. This requires minimum maintenance and human intervention at a fraction of the cost compared to presently available commercial solutions. A pilot instrumentation in the high seismicity area of Lefkas was implemented, in order to collect acceleration data from a significant seismic event and eventually produce explicit seismic hazard maps using custom developed software. 
The experience acquired from the free-field installation and maintenance of the device network has uncovered a few issues that need to be addressed in next hardware/software versions, such as the implementation of RMS triggering. Moreover, there is always the prospect to incorporate future low-cost MEMS sensors with higher accuracy and lower noise levels, as well as to further compact the physical size of the device and simplify its manufacturing process, which will eventually reduce its production cost. Even at their present stage, the developed devices make economically feasible the explicit creation of seismic hazard maps from dense instrumentation arrays, as well as the possibility to validate and calibrate the theoretical methodologies and assumptions used in areas where typical microzonation studies have been performed.

\section{Acknowledgements}

The research project is implemented within the framework of the Action "Supporting Postdoctoral Researchers" of the Operational Program "Education and Lifelong Learning" (Action's Beneficiary: General Secretariat for Research and Technology) and is co-financed by the European Social Fund (ESF) and the Greek State.

\section{References}

[1] Seed, H. B. and Schnabel, P. B. Soil and geological effects on site response during earthquakes. Proc. of the $1^{\text {st }}$ International Conference on Microzonation for Safer Construction - Research and Application, Vol. I, pp. 61-74, 1972.

[2] Avery, H.R., Berrill, J.B. and Dewe, M.B. Design and development of a low-cost, high-performance, strong-motion accelerograph. Proc. of the 2004 NZSEE Conference, New Zealand, 2004.

[3] Kim, S., Pakzad, S., Culler, D.E., Demmel, J., Fenves, G., Glaser, S. and Turon, M. Health monitoring of civil infrastructures using wireless sensor networks, Technical Report No. UCB/EECS-2006-21, Electrical Engineering and Computer Sciences, University of California, Berkeley, 2006.

[4] Picozzi, M., Milkereit, C., Parolai, S., Jäckel, K.H., Veit, I., Fischer, J., Zschau, J. GFZ Wireless Seismic Array (GFZ-WISE), a Wireless Mesh Network of Seismic Sensors: New Perspectives for Seismic Noise Array Investigations and Site Monitoring. Sensors, Vol. 10(4), pp. 3280-3304, 2010.

[5] Wald, D. J., Quitoriano, V., Heaton, T.H. \&, Kanamori, H., Relationships between Peak Ground Acceleration, Peak Ground Velocity and Modified Mercalli Intensity in California. Earthquake Spectra, 15, pp. 557-564, 1999.

[6] Papanikolaou, V.K. \& Karakostas, C.Z., A low cost MEMS-based accelerograph. Experimental Vibration Analysis for Civil Engineering Structures (EVACES'13), Ouro Preto, Brazil, 2013. 